\title{
A steady, purely azimuthal flow model for the Antarctic Circumpolar Current
}

\author{
Ronald Quirchmayr $^{1}{ }_{(\mathbb{D}}$
}

Received: 21 July 2017 / Accepted: 4 September 2017 / Published online: 8 September 2017

(C) The Author(s) 2017. This article is an open access publication

\begin{abstract}
We consider a steady, purely azimuthal eddy viscosity flow model for the Antarctic Circumpolar Current and derive an exact formula for the solution.
\end{abstract}

Keywords Geophysical flows - Ocean dynamics · Eddy viscosity $\cdot f$-plane approximation

Mathematics Subject Classification 86A05 - 35Q35

\section{Introduction}

The Antarctic Circumpolar Current (ACC) flows around Antarctica at latitudes between $40^{\circ}$ to $60^{\circ}$ without meridional boundaries and thereby completely encircles the polar axis towards East. It is the strongest current in the World Ocean and is primarily driven by the wind stress $[12,18]$. Interesting new wave tyes, which may be important to the dynamics of Southern Ocean currents like the ACC were recently derived in [7]. An exact, steady and purely azimuthal model for the ACC in spherical coordinates without eddy viscosity was introduced in [5]. Furthermore we refer

\section{Communicated by A. Constantin.}

The support of the Austrian Science Fund (FWF), Grant W1245, and the European Research Council, Consolidator Grant No. 682537 is acknowledged.

$凶$ Ronald Quirchmayr

ronaldq@kth.se

1 Department of Mathematics, KTH Royal Institute of Technology, Lindstedtsvägen 25, 10044 Stockholm, Sweden 
the reader to [10] for the analysis of an ACC model, which establishes a connection between the free surface and the pressure.

In this paper we consider a purely azimuthal divergence free flow, which is governed by the incompressible Euler equations in the $f$-plane approximation near the 45 th parallel south. The action of mesoscale eddies that transfer the surface stress to the bottom (see [12]) is taken into account by adding an eddy-viscosity term to the system. Furthermore we assume the presence of a constant wind stress and a constant atmospheric pressure on the ocean surface. On the flat bottom we impose a no-slip boundary condition. This system can be solved exactly and the solution is written in terms of the viscosity function, cf. Sect. 3.

\section{The model}

As a starting point we consider the $f$-plane approximation of Euler's equations for three-dimensional divergence-free ocean flows in the region of the ACC. Generally, for a fixed specified polar angle $\theta$, where $\theta$ takes values between $-\pi / 2$ and $\pi / 2$ (e.g. we have that $\theta=0$ along the Equator, $\theta=-\pi / 2$ at the South Pole and $\theta=\pi / 2$ at the North Pole) the $f$-plane approximation is given by

$$
\begin{aligned}
u_{t}+u u_{x}+v u_{y}+w u_{z}+2\left(\Omega^{y} w-\Omega^{z} v\right) & =-\frac{1}{\rho} P_{x} \\
v_{t}+u v_{x}+v v_{y}+w v_{z}+2\left(\Omega^{z} u-\Omega^{x} w\right) & =-\frac{1}{\rho} P_{y} \\
w_{t}+u w_{x}+v w_{y}+w w_{z}+2\left(\Omega^{x} v-\Omega^{y} u\right) & =-\frac{1}{\rho} P_{z}-g \\
u_{x}+v_{y}+w_{z} & =0,
\end{aligned}
$$

where

$$
\Omega^{x}=0, \quad \Omega^{y}=\Omega \cos \theta, \quad \Omega^{z}=\Omega \sin \theta .
$$

Here $t$ denotes the time and $x, y, z$ denote the directions of increasing azimuth, latitude and vertical elevation respectively. Similarly we denote by $u=u(x, y, z, t)$, $v=v(x, y, z, t)$ and $w=w(x, y, z, t)$ the velocity components of the flow field in direction of increasing azimuth, latitude and elevation. Furthermore $P=P(x, y, z, t)$ denotes the pressure field. The constant $\Omega \approx 7.29 \cdot 10^{-5} \mathrm{rad} \mathrm{s}^{-1}$ denotes the rotational speed of the Earth around the polar axis and $g \approx 9.81 \mathrm{~m} \mathrm{~s}^{-2}$ is the gravitational acceleration. The fluid density $\rho$ is taken to be constant, since there is low stratification in the Polar region $[9,18]$.

For modeling the ACC we take $\theta=-\pi / 4$, which corresponds to the 45th parallel south. Furthermore we restrict our considerations to the special case of purely azimuthal flows, i.e. we assume that

$$
v=0 \text { and } w=0 .
$$


Thus (2.1) reduces to the linear system

$$
\begin{aligned}
0 & =-\frac{1}{\rho} P_{x} \\
-\sqrt{2} \Omega u & =-\frac{1}{\rho} P_{y} \\
-\sqrt{2} \Omega u & =-\frac{1}{\rho} P_{z}-g \\
u_{x} & =0 .
\end{aligned}
$$

In order to include the transfer of the wind-generated surface stress to the bottom, which is due to the presence of mesoscale eddies [12], we add a viscosity term of the form $\left(v u_{z}\right)_{z}$ to (2.3) similarly as in [14] in the context of equatorial flows. The classical model of uniform eddy viscosity is due to [17]. We follow the more realistic approach with a depth dependent viscosity function as it was introduced in [8]. For solutions of the equatorial current system and their qualitative properties under the assumption of depth-dependent eddy viscosity we refer to $[3,13,16]$ and the references therein.

Furthermore we add an a priori unknown forcing term $F$ to (2.4), cf. Remark 1, which will be determined in the analysis in Sect. 3. The viscosity function $v=v(z)$, which is assumed to be smooth with $|\nu|>\delta$ for some $\delta>0$, only depends on the depths of water [12], whereas $F$ may depend on both $y$ and $z$. (It is clear that $F$ is independent of $x$.) Thus in summary we end up with the following system:

$$
\begin{aligned}
0 & =-\frac{1}{\rho} P_{x}+\left(\nu u_{z}\right)_{z} \\
-\sqrt{2} \Omega u & =-\frac{1}{\rho} P_{y}+F \\
-\sqrt{2} \Omega u & =-\frac{1}{\rho} P_{z}-g \\
u_{x} & =0 .
\end{aligned}
$$

Before introducing the boundary conditions for $u$ and $P$, we set

$$
\tau=\tau(y, z):=\rho \nu u_{z},
$$

which is referred to as wind stress.

Let $d$ denote the depth of water, which is taken to be constant, i.e. we assume a flat ocean bed. The water surface is considered to be flat, being located at $z=0$. Thus the fluid domain consists of the layer $\mathcal{D}=\mathbb{R}^{2} \times[-d, 0] \subseteq \mathbb{R}^{3}$. On the boundary of $\mathcal{D}$, which consists of the bottom $\{z=-d\}$ and the surface $\{z=0\}$, we impose the following conditions:

$$
\begin{array}{rll}
P=P_{\mathrm{atm}} & \text { on } & z=0 \\
\tau=\tau_{0} & \text { on } & z=0
\end{array}
$$




$$
u=0 \quad \text { on } \quad z=-d .
$$

Here $P_{\text {atm }}$ denotes the constant atmospheric pressure at sea level and $\tau_{0}$ is a constant related to the wind stress at the surface of the ocean.

Remark 1 We note that the usage of the forcing term $F$ is necessary to ensure the existence of a nontrivial flow: otherwise we would end up with the relation $u_{y}=u_{z}$ holding within the entire fluid domain. To see this we first differentiate (2.8) with respect to $z$. Next we differentiate (2.9) with respect to $y$ and combine the respective outcomes. If we assume that $F=0$, we get that $u_{y}=u_{z}$ and the no-slip boundary condition (2.14) implies that $u=0$ throughout $\mathcal{D}$.

Our assumption that the wind-stress at the surface $\tau_{0}$ is independent of both $x$ and $y$ simplifies the model and can not be justified globally, since the wind system in the region of the ACC is rather complex. In fact, the ACC is - in contrast to most other ocean currents - not a single flow, but a fragmented system of strong, regionally bounded jets [15]. Locally, within such a jet, it is reasonable to take $\tau_{0}$ to be constant; cf. the real-time animations of ocean currents and winds on https://earth.nullschool. net/. To get a rough global picture, we may take an average value for $\tau_{0}$.

Let us furthermore emphasize that the linear nature of system (2.7)-(2.10) does not stem from approximations. This is a consequence of the reasonable assumption of a purely azimuthal flow satisfying (2.2).

\section{An exact solution}

In this section we provide an exact formula for the solution of the system (2.7)(2.10), i.e. formulas for the horizontal velocity $u$ and the pressure $P$. These results are summarized in the following theorem.

Theorem 1 The solution of system (2.7)-(2.10) with the boundary conditions (2.12)(2.14) incorporated is given by

$$
u=u(z)=\frac{\tau_{0}}{\rho} \int_{-d}^{z} \frac{\mathrm{d} s}{v(s)}
$$

and

$$
P=P(z)=\sqrt{2} \Omega \rho\left[\int_{-d}^{z} u(s) \mathrm{d} s-\int_{-d}^{0} u(s) \mathrm{d} s\right]-\rho g z+P_{\mathrm{atm}}
$$

for $-d \leq z \leq 0$. The forcing term $F$ satisfies

$$
F=F(z)=-\sqrt{2} \Omega u(z), \quad-d \leq z \leq 0 .
$$

Remark 2 We illustrate the velocity profile of the flow field for a particular viscosity function $v$ in Fig. 1. The horizontal velocity $u$ is generally strictly monotonous, since $v$ does not change sign by assumption, cf. Sect. 2 . 


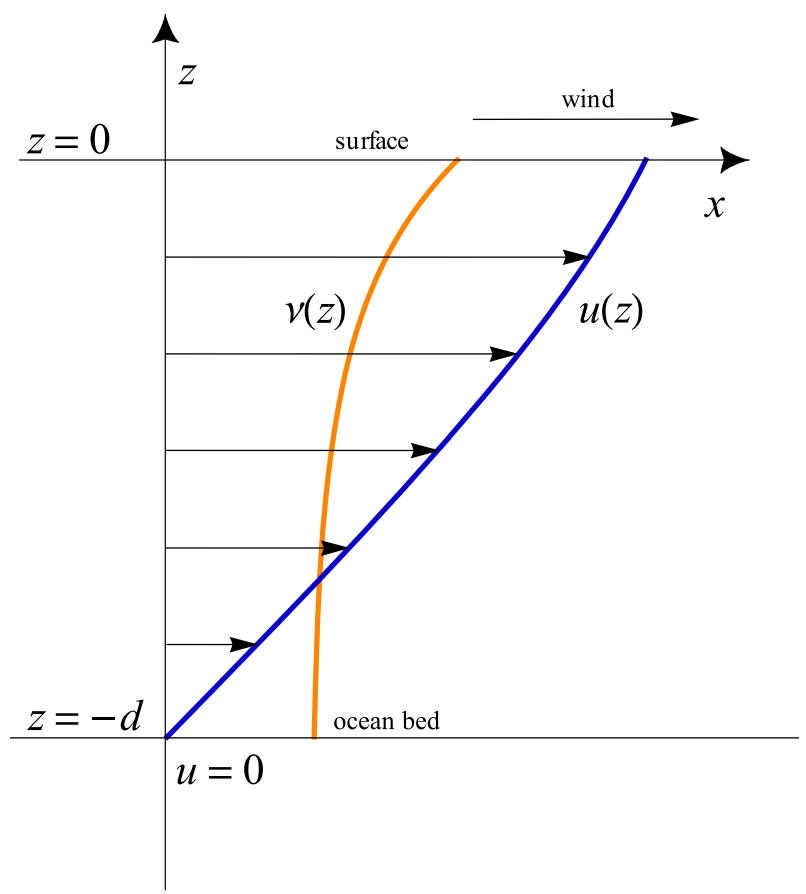

Fig. 1 The viscosity function $v$ (orange) in this plot decreases exponentially with depth. The corresponding solution $u$ (blue) decreases monotonically with depth

The formulas (3.1) and (3.2) in particular reveal that we are actually dealing with a two-dimensional model.

Proof of Theorem 1 By applying $\partial_{x}$ on (2.9) and employing (2.10) we obtain that

$$
P_{x z}=0 \text { in } \mathcal{D} \text {. }
$$

We infer from (2.7) by differentiation with respect to $z$ that

$$
0=-\rho^{-1} P_{x z}+\left(v u_{z}\right)_{z z} \text { in } \mathcal{D} \text {. }
$$

Thus, by (3.4) we have that

$$
0=\left(v u_{z}\right)_{z z} \text { in } \mathcal{D}
$$

From (3.6) and (2.10) we obtain via integration with respect to $z$ that

$$
v u_{z}=A(y) z+B(y) \text { in } \mathcal{D},
$$

and hence

$$
\tau(y, z)=\rho(A(y) z+B(y)) \text { in } \mathcal{D}
$$


due to (2.11). By employing the boundary condition (2.13) we infer that

$$
\tau(y, 0)=\rho B(y)=\tau_{0} \text { for all } y \in \mathbb{R} .
$$

Thus $B$ is independent of $y$ and takes the constant value

$$
B=\tau_{0} / \rho .
$$

By using (3.7) and (2.7) we obtain

$$
\left(v u_{z}\right)_{z}=A(y)=\rho^{-1} P_{x} \text { in } \mathcal{D},
$$

and integration with respect to $x$ yields

$$
\rho^{-1} P=A(y) x+C(y, z) \text { in } \mathcal{D} \text {. }
$$

Since

$$
\rho^{-1} P_{y}=A^{\prime}(y) x+C_{y}(y, z)=\sqrt{2} \Omega u+F(y, z),
$$

where the later expression is independent of $x$, we have that $A^{\prime}(y)=0$, hence

$$
A(y)=A_{1} y+A_{2} \quad \text { with } \quad A_{1,2} \in \mathbb{R}
$$

Since $\left(A_{1} y+A_{2}\right) x+C(y, 0)=\rho^{-1} P_{\text {atm }}$ for all $x, y \in \mathbb{R}$ according to (3.12), (3.14) and (2.12), we conclude that

$$
A_{1}=A_{2}=0 \text { and } C(y, 0)=\rho^{-1} P_{\text {atm }} \text { for all } y \in \mathbb{R} .
$$

In particular we get that

$$
P_{x}=0 \text { in } \mathcal{D} \text {. }
$$

By combining (3.7), (3.10), (3.14), (3.15) and (2.14) we obtain that $u$ is independent of $y$ and satisfies (3.1).

In view of (2.9) we see that $\rho^{-1} P_{z}=\sqrt{2} \Omega u(z)-g$ in $\mathcal{D}$ and integration with respect to $z$ gives

$$
P=P(z)=\sqrt{2} \Omega \rho \int_{-d}^{z} u(s) \mathrm{d} s-\rho g z+C(y) \text { in } \mathcal{D}
$$

Due to the boundary condition (2.12) we infer that the constant of integration $C(y)=$ $C$ in (3.17) is independent of $y$ and satisfies

$$
C=-\sqrt{2} \Omega \rho \int_{-d}^{0} u(s) \mathrm{d} s+P_{\mathrm{atm}}
$$


hence $P$ is given by (3.2).

We finally deduce from (2.8) that $F$ satisfies (3.3), since $P$ is independent of the $y$-coordinate.

Remark 3 In contrast to equatorial flows, where stratification plays a dominant role and leads to two-layer models (see e.g. $[3,4,6,13,16]$ ), there is almost no stratification in the Southern Ocean $[9,18]$. Therefore we considered a one-layer model for describing the dynamics of the ACC, cf. Sect. 2. Let us note that the velocity profile of the solution $u$ of system (2.7)-(2.14) (cf. Fig. 1) differs strongly from stratified flows in equatorial regions like the Equatorial Undercurrent, where $u$ changes its sign (i.e. the direction of the flow gets reversed) at a certain depth between the surface and the thermocline that separates the two layers of different fluid density.

Remark 4 Let us finally point out another difference between equatorial flows and flows in the Southern Ocean like the ACC. While surface waves near the Equator are generally not higher than a few meters (see the discussion in [1]), there exist surface waves in the Southern Ocean having a wave height up to $30 \mathrm{~m}$, cf. [19]. Investigations of such large surface waves and their interactions with underlying currents could thus be of particular interest in the near future. Another interesting question concerns the stability of waves. In [2] it was shown that instability of certain waves in equatorial regions does occur. On the other hand, short-wavelength stability results for certain equatorial flows are available in [11]. Similar stability results in the context of Southern Ocean currents would be of great interest.

Acknowledgements The author is grateful for helpful comments from the referee.

Open Access This article is distributed under the terms of the Creative Commons Attribution 4.0 International License (http://creativecommons.org/licenses/by/4.0/), which permits unrestricted use, distribution, and reproduction in any medium, provided you give appropriate credit to the original author(s) and the source, provide a link to the Creative Commons license, and indicate if changes were made.

\section{References}

1. Constantin, A.: An exact solution for equatorially trapped waves. J. Geophys. Res. Oceans 117, C05029 (2012)

2. Constantin, A., Germain, P.: Instability of some equatorially trapped waves. J. Geophys. Res. Oceans 118, 2802-2810 (2013)

3. Constantin, A., Johnson, R.S.: The dynamics of waves interacting with the equatorial undercurrent. Geophys. Astrophys. Fluid Dyn. 109(4), 311-358 (2015)

4. Constantin, A., Johnson, R.S.: An exact, steady, purely azimuthal equatorial flow with a free surface. J. Phys. Oceanogr. 46, 1935-1945 (2016)

5. Constantin, A., Johnson, R.S.: An exact, steady, purely azimuthal flow as a model for the antarctic circumpolar current. J. Phys. Oceanogr. 46, 3585-3594 (2016)

6. Constantin, A., Johnson, R.S.: A nonlinear, three-dimensional model for ocean flows, motivated by some observations of the Pacific Equatorial Undercurrent and thermocline. J. Phys. Oceanogr. 46, 3585-3594 (2016)

7. Constantin, A., Monismith, S.G.: Gerstner waves in the presence of mean currents and rotation. Phys. Fluids 29, 056604 (2017)

8. Cronin, M.F., Kessler, W.S.: Near-surface shear flow in the tropical Pacific cold tongue front. J. Phys. Oceanogr. 39, 1200-1215 (2009) 
9. Firing, Y.L., Chereskin, T.K., Mazloff, M.R.: Vertical structure and transport of the Antarctic Circumpolar Current in Drake Passage from direct velocity observations. J. Geophys. Res. 116, C08015 (2004)

10. Hsu, H.C., Martin, C.I.: On the existence of solutions and the pressure function related to the Antarctic circumpolar current. Nonlinear Anal. 155, 285-293 (2017)

11. Ionescu-Kruse, D., Martin, C. I.: Local Stability for an exact steady purely azimuthal equatorial flow. J. Math. Fluid Mech., to appear. doi:10.1007/s00021-016-0311-4

12. Ivchenko, V.O., Richards, K.J.: The dynamics of the Antarctic circumpolar current. J. Phys. Oceanogr. 26, 753-774 (2012)

13. Martin, C.I.: Dynamics of the thermocline in the equatorial region of the Pacific ocean. J. Nonlinear Math. Phys. 22, 516-522 (2015)

14. McCreary, J.P.: Modeling equatorial ocean circulation. Annu. Rev. Fluid Mech. 17, 359-409 (1985)

15. Olbers, D., Borowski, D., Völker, C., Wolff, J.-O.: The dynamical balance, transport and circulation of the Antarctic Circumpolar Current. Antarctic Sci. 16(4), 439-470 (2004)

16. Quirchmayr, R.: On the existence of benthic storms. J. Nonlinear Math. Phys. 22, 540-544 (2015)

17. Stommel, H.: Wind drift near the equator. Deep Sea Res. 6, 298-302 (1960)

18. Tomczak, M., Godfrey, J.S.: Regional Oceanography: An Introdution. Pergamon Press, Oxford (1994)

19. Walton, D.W.H.: Antarctica: Global Science from a Frozen Continent. Cambridge University Press, Cambridge (2013) 